\title{
Numerical Simulation of Particle Motions in Cascade Impactor and Human Respiratory System
}

\author{
Shuji OHSAKI*, Ryosuke Mitani, Saki FuJIWARA, Hideya NAKAMURA, and Satoru WATANO \\ Department of Chemical Engineering, Osaka Prefecture University, 1-1 Gakuen-cho, Naka-ku, Sakai, Osaka 599-8531, Japan
}

\begin{abstract}
Dry powder inhalations (DPIs) have gathered attention as a treatment for respiratory diseases due to the large effective absorption area in a human lung. A cascade impactor is generally used to investigate the inhalation performance of DPIs. For the improvement of the efficiency of DPIs, understanding the particle motion and deposition behavior in the human lung and the cascade impactor is required. In the present study, computer simulations were conducted to calculate the particle motion and deposition behavior in the human lung and the cascade impactor. As simulation methods, a coupling model of a computational fluid dynamics and a discrete phase method (CFD-DPM) and a coupling model of a CFD and a discrete element method (CFD-DEM) were used. The CFD-DEM simulation could reproduce the experimental particle deposition behavior in the cascade impactor, although it was difficult by the CFD-DPM simulation. Furthermore, the calculation results using the CFD-DEM simulation quantitatively demonstrated the higher particle reachability into the simple lung model when smaller particles were used. It was found that the CFD-DEM simulation is a powerful tool to calculate the particle motion and deposition behavior in the cascade impactor and human lung.
\end{abstract}

\section{Introduction}

Dry powder inhalations (DPIs) are a dosage form for delivering powdered medicine to the human lungs by an inhaled airflow of patients, and it is mainly used as a treatment for respiratory diseases such as bronchial asthma and chronic obstructive lung disease (Islam and Gladki, 2008). Main factors affecting the deposition site in the human respiratory system are the particle properties (size, density, and shape). The design of DPIs for the effective transportation in the human respiratory system has been investigated, in which a cascade impactor is generally used to evaluate the particle reachability into the human lung (Kaialy et al., 2012). Understanding particle motion and deposition behavior in not only a respiratory system but also a cascade impactor is necessary to improve the efficiency of DPIs. The present study proposed the usage of a coupling model of a computational fluid dynamics and a discrete phase method (CFD-DPM) and a coupling model of a CFD and a discrete element method (CFD-DEM). A cascade impactor model and a simple lung model were constructed to calculate the behavior of the fluid and particle transportation.

\section{Simulation and Experimental Method}

\subsection{Model geometry}

Figure 1 shows the geometry of the cascade impactor and simple lung models. They were created using a commercial software (ANSYS Design Modeler Version 17.2, ANSYS Inc.). The cascade impactor model (Figure 1a) has 8 stages (Stage $0-7$ ). At the bottom of each stage, the collection plates with the holes which have different numbers (210-880) and diameters $(0.25-1.2 \mathrm{~mm})$ were located. In the simple symmetrical lung model (Figure 1(b)), the bronchi were maximally branched to six and narrowed with branching $(12-100 \mathrm{~mm})$. The model geometry was divided into six zones. Zone- 6 included the end of the simple lung model geometry. The calculation cells were prepared using a cell creation

(a)

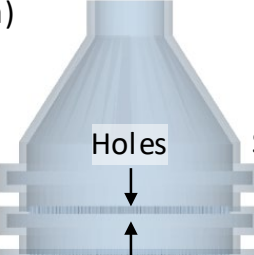

Collection plate

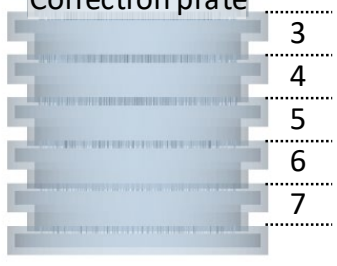

Figure 1. Schematic of (a) the cascade impactor model and (b)

the simple lung model

(b)

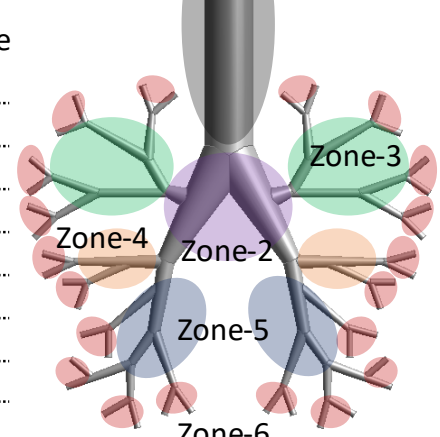

Zone-6

\footnotetext{
Shuji Ohsaki: ohsaki@chemeng.osakafu-u.ac.jp
} 
software (ANSYS Meshing Version 17.2, ANSYS Inc.). The cell numbers of the cascade impactor and simple lung models were approximately 3,080,000 and 220,000, respectively.

\subsection{Simulation model}

The fluid dynamics in the cascade impactor and simple lung models was calculated using the CFD method. The calculation of the particle motions was conducted by using the DPM or DEM.

The governing equations for incompressible fluids are the continuity equation and the Navier-Stokes equation (Eqs. 1 and 2).

$$
\begin{aligned}
& \nabla \cdot \boldsymbol{u}_{\mathrm{f}}=0 \\
& \frac{\partial \rho_{\mathrm{f}} \boldsymbol{u}_{\mathrm{f}}}{\partial t}=-\nabla \cdot\left(\rho_{\mathrm{f}} \boldsymbol{u}_{\mathrm{f}} \boldsymbol{u}_{\mathrm{f}}\right)-\nabla p+\nabla \cdot\left(\tau_{\mathrm{f}}\right)+\rho_{\mathrm{f}} \boldsymbol{g}
\end{aligned}
$$

where $\boldsymbol{u}_{\mathrm{f}}, \rho_{\mathrm{f}}, p$, and $\tau_{\mathrm{f}}$ indicate fluid velocity vector, fluid density, fluid pressure, and stress-strain tensor, respectively.

The CFD-DPM simulation was conducted using the Euler-Lagrange method. In this method, the particle motions were described as

$$
\frac{\mathrm{d} \boldsymbol{u}_{\mathrm{p}}}{\mathrm{d} t}=\frac{18 \mu_{\mathrm{f}}}{\rho_{\mathrm{p}} d_{\mathrm{p}}^{2}} \frac{C_{D} R e_{\mathrm{p}}}{24}\left(\boldsymbol{u}_{\mathrm{p}}-\boldsymbol{u}_{\mathrm{f}}\right)+g\left(1-\frac{\rho_{\mathrm{f}}}{\rho_{\mathrm{p}}}\right)
$$

where $\boldsymbol{u}_{\mathrm{p}}, \mu_{\mathrm{f}}, \rho_{\mathrm{p}}$, and $d_{\mathrm{p}}$ indicate particle velocity vector, fluid viscosity, particle density, and particle size, respectively. $C_{D}$ and $R e_{\mathrm{p}}$ are the drag coefficient and particle Reynolds number, respectively, and they are described by

$$
\begin{aligned}
& C_{D}=\frac{K_{1}}{R e_{\mathrm{p}}}+\frac{K_{2}}{R e_{\mathrm{p}}^{2}}+K_{3} \\
& R e_{\mathrm{p}}=\frac{\rho_{\mathrm{f}} d_{\mathrm{p}}\left|\boldsymbol{u}_{\mathrm{p}}-\boldsymbol{u}_{\mathrm{f}}\right|}{\mu_{\mathrm{f}}}
\end{aligned}
$$

where $K_{1}, K_{2}$, and $K_{3}$ depending on $R e_{\mathrm{p}}$ were used based on the Morsi-Alexander model (Morsi and Alexander, 1972).

The DEM-CFD simulation was conducted based on the local volume average method (Tsuji et al., 1993). In this method, the governing equations of fluid phase use the local void fraction. The drag force exerted on the particle was calculated using the Schiller-Naumann model (Shiller and Naumann, 1935). In the DEM calculation, a Voight model was used as the contact force on the particles, and the adhesion force based on the JKR theory (Johnson et al., 1971) was applied.

\subsection{Calculation condition}

A thermal fluid analysis software (ANSYS Fluent Ver. 17.2, ANSYS, Inc.) and a DEM simulation software (EDEM 2018, DEM Solutions Co. Ltd.) were used to perform the CFD and DEM simulations. The fluid phase was calculated as a nonsteady calculation of incompressible fluid. The standard $k-\omega$ model was applied as a turbulence model. A SIMPLE method (Patankar and Spalding, 1972) was used for the numerical analysis. In the CFD-DPM simulation using the cascade impactor model, the particle size and the flow rate were set to be $100 \mu \mathrm{m}$ or $10 \mu \mathrm{m}$ and 28.3 $\mathrm{L} / \mathrm{min}$, respectively. In the case of the CFD-DEM simulations, the particles with a size distribution (median size is $97.2 \mu \mathrm{m}$ and standard division is $23.7 \mu \mathrm{m}$ ) was used to calculate the particle motion in the cascade

\begin{tabular}{|c|c|c|}
\hline Fluid pressure & 101.325 & $\mathrm{kPa}$ \\
\hline Fluid density & 1.225 & $\mathrm{~kg} / \mathrm{m}^{3}$ \\
\hline Fluid viscosity & $1.789 \times 10^{-5}$ & $\mathrm{~Pa} \cdot \mathrm{s}$ \\
\hline Particle size & $5-20$ & $\mu \mathrm{m}$ \\
\hline Particle density & 720 & $\mathrm{~kg} / \mathrm{m} 3$ \\
\hline Coefficient particle-particle & 0.5 & - \\
\hline of restitution particle-lung & 0.9 & - \\
\hline Coefficient particle-particle & 0.5 & - \\
\hline of static friction particle-lung & 0.7 & - \\
\hline Coefficient particle-particle & 0.01 & - \\
\hline of rolling friction particle-lung & 0.7 & - \\
\hline Poisson's ratio of particle & 0.3 & - \\
\hline Poisson's ratio of lung & 0.3 & - \\
\hline Young's modulus of particle & $1 \times 10^{8}$ & $\mathrm{~Pa}$ \\
\hline Shear modulus of lung & $5 \times 10^{5}$ & $\mathrm{~Pa}$ \\
\hline Surface energy & 0.5 & $\mathrm{~J} / \mathrm{m} 2$ \\
\hline
\end{tabular}
impactor model. The detailed calculation conditions for the CFD-DEM simulation using the simple lung model are summarized in Table 1.

Table 1. Calculation conditions for CFD-DEM simulations.

\subsection{Experiment}

A cascade impactor (AN-200, Tokyo Dylec Corp.) was used to investigate the deposition behavior of mannitol particles experimentally (NONPAREIL-108®), Freund Corp.). The median size and standard division of mannitol particles were $90.0 \mu \mathrm{m}$ and $11.3 \mu \mathrm{m}$, respectively. The mannitol particles $(20 \mathrm{mg})$ were introduced in the capsule (HPMC capsule, Qualicas Co., Ltd.), followed by setting in an inhalation device (Jethaler ${ }^{\circledR}$, Hitachi Automotive Systems, Ltd.). The mannitol particles were inhaled into the cascade impactor via the device at $28.3 \mathrm{~L} / \mathrm{min}$ for $5 \mathrm{~s}$. The deposition ratios on each stage were calculated as a mass ratio of particles arriving at each stage to the total particle mass.

\section{Results and Discussion}

\subsection{Fluid dynamics in the cascade impactor}

Figure 2(a) shows the fluid velocity contours in Stages 0 and 1 (cross section at $y=0$ ). The inlet air passed through the holes and hit the collection plate, followed by the transportation to the lower stages through the gap around the collection plate. Under the collection stage, the air flow formed a vortex. The particles moved along this air flow and deposited on the collection plates. This fluid dynamics was observed in each stage. Figure 2(b) indicates the average airflow velocity through the holes 
(a)

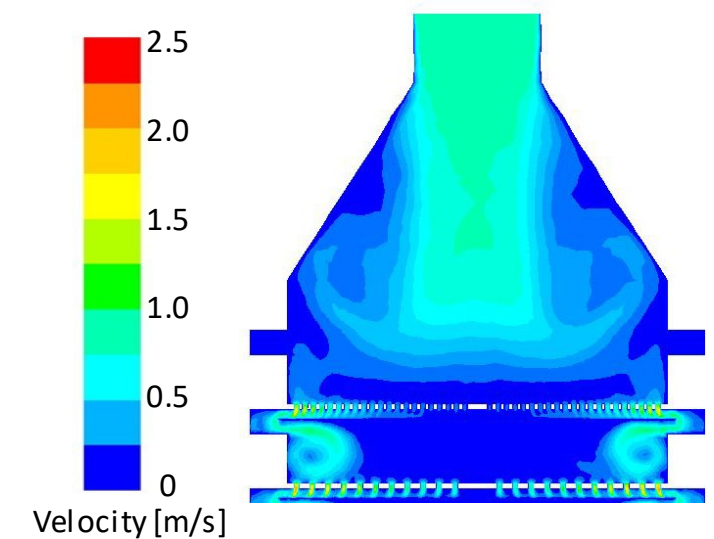

(b)

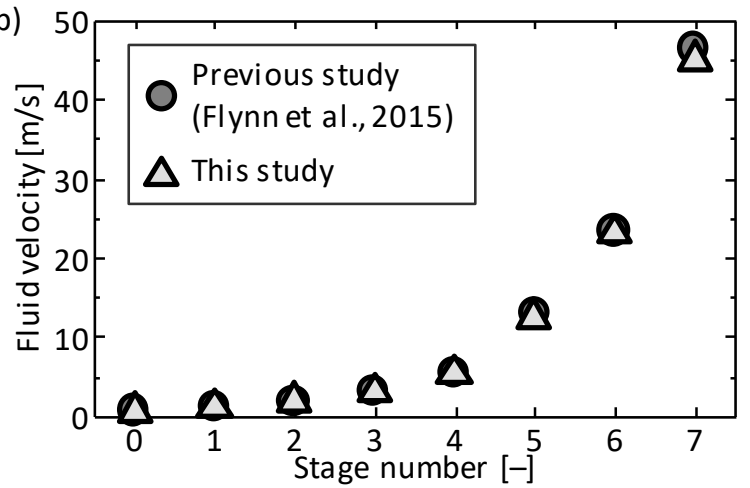

Figure 2. (a) Fluid velocity contours in Stages 0 and 1 in the cascade impactor model and (b) average air flow velocity through the holes at different stage

at different stages. The fluid velocity increased at the lower stages. The fluid velocities at Stage 7 were found to be about 10 times faster than that of Stage 0 . This is attributed to the smaller holes at lower stages. Also, the simulated flow velocities in this study were corresponding to the reported values (Flynn et al., 2015). The validity of the CFD simulation method, including the constructed model and meshing, was confirmed.

\subsection{Particle deposition behavior in the cascade impactor}

The particle motions in the cascade impactor model were calculated using the CFD-DPM coupling model. Figure 3 compares the particle deposition positions of $100 \mu \mathrm{m}$ and $10 \mu \mathrm{m}$. The particle reachability to Stage 6 and 7 of $100 \mu \mathrm{m}$ was higher than that of $10 \mu \mathrm{m}$. However, the smaller particles reach the lower stages theoretically. Therefore, the calculated results using the CFD-DPM

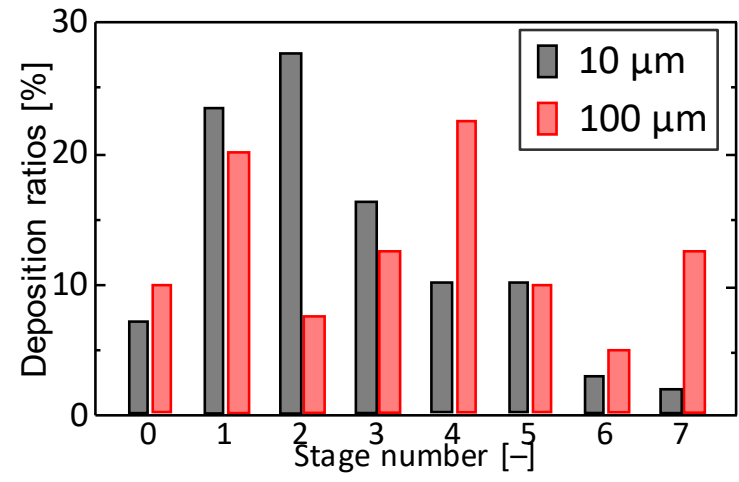

Figure 3. Particle deposition ratios of $100 \mu \mathrm{m}$ and $10 \mu \mathrm{m}$
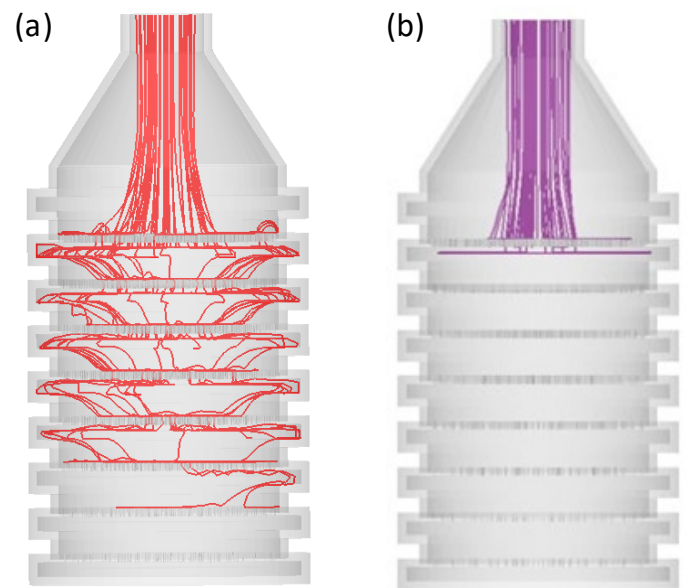

Figure 4. Particle motion trajectories (a) without and (b) with consideration of adhesion

model was found to disagree with the actual particle motions. This might be attributed to the assumption of the mass point in the CFD-DPM model. In the mass point assumption, the collision of particle-particle and particle-wall could be hardly considered.

The CFD-DEM coupling model was used to calculate the particle motions in the cascade impactor. The particle motion trajectories without/with consideration of adhesion force based on the JKR theory are shown in Figure 4. Although particles reached the lower stages in the case of calculation without adhesion (Figure 4a), they were deposited on the upper stages when considering the adhesion (Figure $4 \mathrm{~b}$ ). These results suggested that not only the inertial force but also the adhesion force affected the particle deposition behavior in the cascade impactor strongly.

Figure 5(a) shows the deposition position in the cascade impactor under different surface energies $\left(\gamma=0-1 \mathrm{~J} / \mathrm{m}^{2}\right)$.
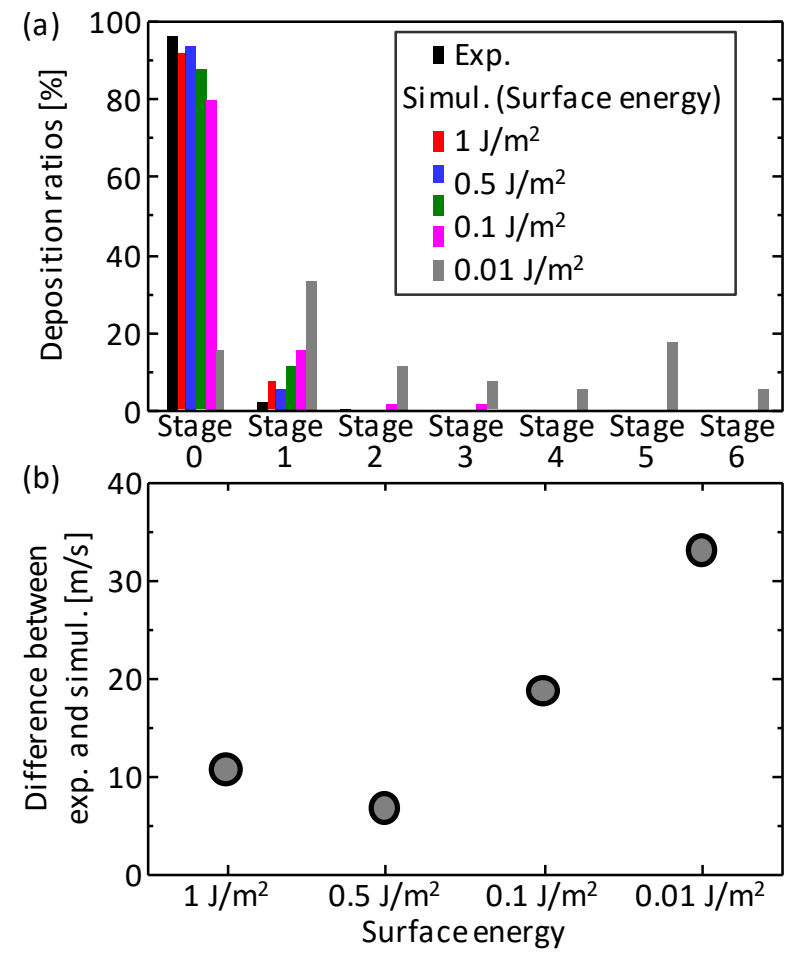

Figure 5. (a) Deposition position in the cascade impactor under the different surface energy and (b) difference between the calculated values and experimental ones 
(a)

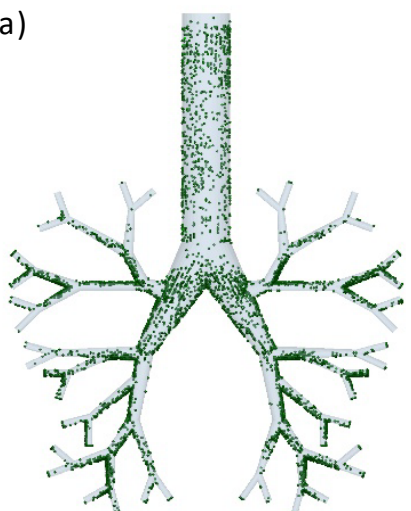

(b)

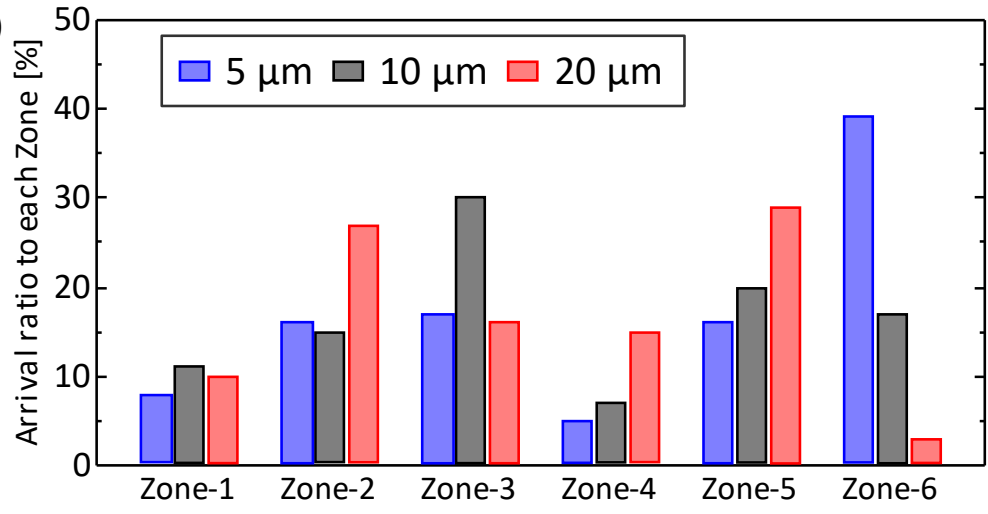

Figure 6. (a) Particle arrival position in the simple lung model and (b) arrival ratios to each zone for different particle size

The deposition ratio on the upper stages increased with the surface energy. Figure 5(b) indicates the difference between the calculated values and experimental ones. The surface energy of $0.5 \mathrm{~J} / \mathrm{m}^{2}$ shows the smallest difference, which suggested that $0.5 \mathrm{~J} / \mathrm{m}^{2}$ was optimum for the surface energy of the particles.

\subsection{Particle reachability into the simple lung model}

The DEM-CFD coupling model was applied to the calculation of particle motions in the simple lung model. Figure 6(a) shows the particle arrival positions in the simple lung model calculated under the particle conditions of $10 \mu \mathrm{m}$ and $720 \mathrm{~kg} / \mathrm{m}^{3}$. The particles were magnified 100 times. The particles introduced from the inlet reached the depth of the simple lung model after approximately $0.2 \mathrm{~s}$. They never moved after adhering on the lung wall. It was found that the arrival ratio to each zone, defined in Figure 1(b), was 11\%, 15\%, 30\%, $7 \%, 20 \%$, and $17 \%$ for Zones-1-6, respectively. Figure 6 (b) shows the arrival ratios to each zone calculated under different particle sizes. The $20 \mu \mathrm{m}$ particles showed high arrival ratios to Zone- 2 and Zone- 5 , while 5 $\mu \mathrm{m}$ particles showed the highest arrival ratio to Zone- 6 . This would be because the large particles transported in the direction of gravity regardless of the fluid dynamics, while the small particle motions agreed with the fluid flow. These results suggested that the smaller particles showed higher particle reachability into the depth of the simple lung model. From the above results, the DEM-CFD calculation using the simple lung model quantitatively demonstrated the particle trajectory and reachability into its depth. Moreover, it is suggested that DEM-CFD model is suitable for analysis of particle motions in the human lung.

\section{Conclusion}

In the present study, the particle motions in the cascade impactor and simple lung models were calculated using the CFD-DPM and CFD-DEM coupling models. The CFD-DPM simulation was not suitable to calculate particle motions in the cascade impactor model due to the mass point assumption. On the other hand, the
CFD-DEM simulation with consideration of adhesion force could successfully perform the experimental particle deposition behaviour in the cascade impactor. Moreover, the DEM-CFD simulation for the simple lung model demonstrated that the smaller particles reached its deeper regions. The present study found that the DEM-CFD simulation was suitable for the analysis of the particle motions in the cascade impactor and human lung models.

\section{Acknowledgments}

This work was partially supported by Hosokawa Powder Technology Foundation.

\section{References}

Flynn, S. J. Z. B.Tong, R. Y. Yang, H. Kamiya, A. B. $\mathrm{Yu}$, and H. K. Chand ; "Computational Fluid Dynamics (CFD) Investigation of the Gas-Solid Flow and Performance of Andersen Cascade Impactor," Powder Technology, 285, 128-137 (2015)

Islam, N. and E. Gladki; "Dry Powder Inhalers (DPIs)-a Review of Device Reliability and Innovation," International Journal of Pharmaceutics, 360, 1-11 (2008)

Johnson, K. L., K. Kendall, and A. D. Roberts; "Surface Energy and the Contact of Elastic Solids, Proceedings of the Royal Society A: Mathematical," Physical and Engineering Sciences, 324, 301-313 (1971)

Kaialy, W., A. Alhalaweh, S. P.Velaga, and A, Nokhodchi; "Influence of Lactose Carrier Particle Size on the Aerosol Performance of Budesonide from a Dry Powder Inhaler," Powder Technology, 227, 74-85 (2012)

Morsi, S. A. and A. J. Alexander; "An Investigation of Particle Trajectories in Two-Phase Flow Systems," Journal of Fluid Mechanics, 55, 193-208 (1972)

Patankar, S. V. and D. B. Spalding; "A Calculation Procedure for Heat, Mass and Momentum Transfer in Three-Dimensional Parabolic Flows," International Journal of Heat and Mass Transfer, 15, 1787-1806 (1972) 
Shiller, L. and A. Naumann; "A Drag Coefficient Correlation," Zeitschrift des Vereins Deutscher Ingenieure, 77, 318-320 (1935)

Tsuji, Y., T. Kawaguchi and T. Tanaka; "Discrete Particle Simulation of Two-Dimensional Fluidized Bed," Powder Technology," 77, 79-87 (1993) 